\title{
Análise do consumo de tabaco e avaliação da capacidade respiratória em estudantes universitários
}

\author{
Analysis of tobacco consumption and respiratory capacity \\ assessment in university students
}

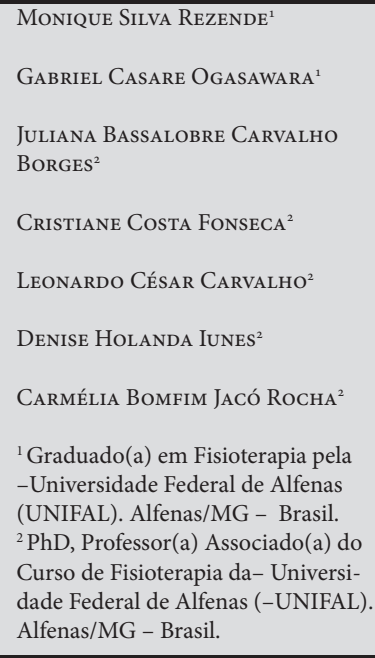

Resumo O tabagismo é considerado a principal causa de morte evitável, sendo um vício comumente iniciado na juventude. Torna-se importante a avaliação das alterações que esse hábito pode causar nessa faixa etária. Objetivo: Identificar o consumo de tabaco e comparar a capacidade respiratória entre estudantes universitários fumantes e não fumantes.

Métodos: Estudo transversal, dividido em duas etapas. Amostra total na primeira etapa com 1.071 estudantes, de ambos os sexos, média de idade de 21,2 anos $( \pm 2,0)$; sendo aplicado um questionário sobre hábitos tabágicos. Na segunda etapa, os indivíduos foram agrupados em tabagistas e não tabagistas. Dentro de cada grupo foram sorteados aleatoriamente 20 indivíduos, 10 de cada sexo, totalizando 40 indivíduos para analisar a capacidade respiratória. $\mathrm{Na}$ avaliação da capacidade respiratória, utilizaram-se as técnicas de cirtometria torácica e manovacuometria (pressões respiratórias máximas, PImax e PEmax).

Resultados: Em 15\% dos alunos verificou-se o hábito de fumar. Com relação à capacidade respiratória, na comparação entre os grupos tabagista e não tabagista, os alunos fumantes apresentaram menores valores significantes, de pressão inspiratória (PImax; $\mathrm{p}<0,001)$, e expiratória máxima (PEmax; $\mathrm{p}<0,001)$. Os valores obtidos por meio da cirtometria torácica também apresentaram diferenças significantes entre os grupos (coeficiente respiratório axilar $[\mathrm{p}<0,001]$; coeficiente respiratório mamilar $[\mathrm{p}<0,001]$ e coeficiente respiratório da $10^{\mathrm{a}}$ costela $\left.[\mathrm{p}<0,001]\right)$. Conclusão: Foram identificados $15 \%$ de usuários de tabaco entre os estudantes da UNIFAL-MG. Em relação à capacidade respiratória desses estudantes tabagistas, quando comparada com não tabagistas, foram evidenciados menores valores de capacidade respiratória nos parâmetros de PImax, PEmax e cirtometrias. Dessa forma, observou-se que o tabaco pode levar a alterações na capacidade respiratória de forma precoce.

Palavras-chave: Técnicas de Diagnóstico do Sistema RespiraTÓRIO. HÁbito de Fumar. EstudANTES.

Abstract Smoking is considered the main cause of preventable death, commonly started in youth. It is important to evaluate the changes that this habit can cause in youth groups.

Objective: Identify tobacco consumption and to compare respiratory capacity among college students smokers and nonsmokers. 
Methods: Cross-sectional study, divided in two stages. In the first stage participated 1071 students, of both sexes, middle age of 21.2 years $( \pm 2.0)$. For this sample was applied a questionnaire of smoking habits. In the second stage, individuals were grouped into smokers and non-smokers. Within each group, 20 subjects were randomly selected, 10 of each sex, totalizing 40 individuals to analyze the respiratory capacity. In the evaluation of the respiratory capacity, the techniques of thoracic cirtometry and manovacuometry (maximum respiratory pressures, MIP and MEP) were used.

Results: Smoking habit was present in $15 \%$ of the students. Regarding respiratory capacity, smoking students had lower values of inspiratory pressure (PImax, $\mathrm{p}<0.001$ ), and maximal expiratory (SMmax, $\mathrm{p}<0.001$ ) in comparison between smoking and non-smoker groups. The values obtained by thoracic cirtometry also showed significant differences between the groups (axillary respiratory coefficient $[\mathrm{p}<0.001]$, mammillary respiratory coefficient $[\mathrm{p}<0.001]$ and respiratory coefficient of the 10th cartilage $[\mathrm{p}<0.001])$.

Conclusion: $15 \%$ of tobacco users were identified among UNIFAL-MG students. Regarding the respiratory capacity of these smokers, when compared to non-smokers, lower values of respiratory capacity were found in the parameters of MIP, MEP and cirtometries. In this way, it was observed that tobacco can lead earlier to changes in respiratory capacity.

Key-words: Diagnostic Techniques. Respiratory System. Smoking. Students.

\section{INTRODUÇÃO}

O tabagismo é o responsável por altos índices de morbimortalidade mundial, sendo considerado a principal causa de morte evitável em todo mundo. A prevalência de fumantes no mundo é de 1,3 bilhão, constituindo um terço da população global. ${ }^{1,2} \mathrm{~A}$ previsão para o ano 2020 é ocorrerem 10 milhões de óbitos em consequência do tabagismo, sendo sete milhões nos países em desenvolvimento. Persistindo essa tendência, no século XXI, eventualmente, ocorrerá um bilhão de óbitos. ${ }^{1}$

O tabaco representa a segunda droga mais consumida no mundo. Um terço da população mundial adulta é fumante e esse hábito aumenta o risco de mortalidade por câncer e por doenças cardiovasculares, cerebrovasculares e respiratórias crônicas. ${ }^{3}$ Os jovens são o público-alvo das estratégias das indústrias do tabaco. Esse investimento tem o intuito de garantir a manutenção de um grande contingente de consumidores. ${ }^{3}$ Estudos demonstram que $80 \%$ dos fumantes iniciam o hábito antes dos 18 anos de idade, apontando uma instalação precoce ${ }^{3,4,5}$ no período de transição entre o ensino mé- dio para o superior. ${ }^{6}$ Isso acontece porque os jovens se encontram em um período de formação de caráter, e isso os torna mais influenciáveis tanto pela mídia quanto pelo grupo social do qual fazem parte. ${ }^{4,5}$

Alguns autores relacionam o uso de tabaco pelos adolescentes a fatores ambientais, sociodemográficos, comportamentais e pessoais; ${ }^{7}$ bem como ao hábito de fumar dos pais, amigos e irmãos com maior idade; baixo rendimento nos estudos; abandono da escola e trabalho remunerado na adolescência. ${ }^{5,8}$ Algumas condições que aumentam a probabilidade da fixação do tabagismo entre os adolescentes são desajuste familiar, separação dos pais e moradia com outras pessoas que não os pais biológicos, baixa autoestima e depressão. Deve-se assinalar, ainda, como facilitador para a iniciação, a limitada percepção entre os adolescentes do risco decorrente do tabagismo. ${ }^{9}$ Outro estudo aponta como fatores de proteção ao tabagismo, a orientação parental sobre o tabagismo, a não exposição ao tabagismo em casa e o conhecimento do tabaco. ${ }^{5}$

Estudos na tentativa de compreender as características de consumo do tabaco e 
o perfil da população de interesse visam auxiliar o desenvolvimento de estratégias que tenham como objetivo aprimorar programas de prevenção existentes em instituições de ensino superior além de formas de incentivar a profilaxia do consumo de cigarros nessa população. ${ }^{10}$

Assim, este estudo teve como objetivo identificar entre os graduandos da Universidade Federal de Alfenas em Minas Gerais (UNIFAL-MG), o consumo de tabaco, além da comparação da capacidade respiratória entre alunos fumantes e não fumantes.

\section{Métodos}

O estudo foi aprovado pelo Comitê de Ética e Pesquisa da Universidade Federal de Alfenas (Protocolo no. 166/2011).

\section{População e amostra}

A população do estudo foi formada por alunos de ambos os sexos, fumantes e não-fumantes matriculados nos cursos de graduação da Universidade Federal de Alfenas-MG. A seleção da amostra foi feita por meio de convite nas salas dos cursos de graduação.

$\mathrm{O}$ estudo foi dividido em duas etapas. $\mathrm{Na}$ primeira, a amostra total foi 1.071 estudantes voluntários, com média de idade de 21,2 \pm 2,0 anos; sendo aplicado um questionário.

$\mathrm{Na}$ segunda etapa, os indivíduos foram agrupados em tabagistas e não tabagistas, dentro de cada grupo foram sorteados aleatoriamente 20 indivíduos, 10 de cada sexo, totalizando 40 indivíduos para analisar a capacidade respiratória.

\section{Critérios de Inclusão e Exclusão}

Como critérios de inclusão, todos os graduandos de ambos os sexos, devidamen- te matriculados na Universidade Federal de Alfenas (UNIFAL-MG), foram convidados a participar do estudo; após esclarecimentos sobre os objetivos da pesquisa, os alunos que concordaram em participar assinaram o Termo de Consentimento Livre e Esclarecido. Foram excluídos estudantes com presença ou histórico de alguma doença pulmonar obstrutiva, fratura de costelas, cirurgias torácicas e abdominais, problemas neurológicos.

\section{Procedimentos de avaliação}

$\mathrm{Na}$ primeira etapa foi aplicado um questionário construído pelos pesquisadores visando obter informações necessárias a respeito do consumo do tabaco, segundo adaptação do Questionário sobre o Consumo do Tabaco da Organização Mundial de Saúde, validado para o Brasil pelo INCA. ${ }^{11}$ Nesse instrumento, são obtidas informações como idade; sexo; com quantos anos experimentou tabaco; se possui o hábito de fumar, se as campanhas do governo sensibilizam a não fumar; o que influenciou a fumar, quantos cigarros fumam por dia; se consome pelo preço ou pela marca e se conhece alguma doença decorrente do hábito de fumar.

$\mathrm{Na}$ segunda etapa, os indivíduos avaliados foram agrupados em tabagistas e não tabagistas, dentro de cada grupo foram sorteados aleatoriamente 20 indivíduos, 10 de cada sexo, totalizando 40 indivíduos para analisar a capacidade respiratória. Na avaliação da capacidade respiratória, foram consideradas as variáveis de expansibilidade torácica e pressões respiratórias máximas. 


\section{Avaliação da expansibilidade torácica}

Para a realização da cirtometria, foi utilizada uma fita métrica da marca Fisio Store $^{\circledast}$ com escala de 0 a 150 centímetros e precisão de 0,1 centímetros. ${ }^{12}$ Foram medidos os perímetros torácicos de cada indivíduo em três regiões: 1) perímetro axilar com a fita métrica passando pelos cavos axilares ao nível da terceira costela; 2) perímetro xifoide, passando sobre o apêndice xifoide ao nível da sétima cartilagem costal e 3) perímetro basal, passando sobre as $10^{\text {a }}$ costelas. ${ }^{12} \mathrm{O}$ ponto de partida para realizar essa medida foi o ponto (0) zero da fita métrica, ou seja, com o sujeito em expiração máxima, em nível de volume residual (VR). Em seguida, realizou-se uma inspiração máxima ao nível da capacidade pulmonar total (CPT) e, posteriormente, na expiração máxima, ao nível do VR, mensurando os resultados por meio da fita métrica, cuja diferença entre as medidas máximas são chamadas de Coeficiente Respiratório (CR). Os testes foram feitos nas três regiões já citadas, com três mensurações em cada região. ${ }^{12}$

\section{Avaliação da pressão respiratória}

As pressões inspiratórias e expiratórias máximas (PImáx e PEmáx), em $\mathrm{cmH}_{2} \mathrm{O}$, foram medidas com um manovacuômetro analógico da marca Comercial Médica, MV120, com intervalo operacional de \pm $120 \mathrm{cmH}_{2} \mathrm{O}$, adaptado para pressões inspiratórias e expiratórias máximas. A PImáx foi medida durante esforço iniciado a partir do VR, enquanto que a PEmáx foi medida a partir da CPT. ${ }^{13} \mathrm{O}$ manômetro foi acoplado a um tubo rígido e a um bocal pelo qual o indivíduo realizou o teste que indicou a força dos músculos inspiratórios, pela pressão máxima exercida na inspiração, e a força dos músculos expiratórios, pela pressão máxima exercida na expiração. ${ }^{13}$

Para medir a pressão inspiratória máxima, o indivíduo permaneceu sentado com os pés apoiados a $90^{\circ}$ no chão, bocal e pinça nasal posicionados. Solicitou-se a realização de dois ou três ciclos respiratórios normais e, após isso, uma expiração completa ao nível de VR. O indivíduo foi orientado a indicar esse momento por meio de um gesto. ${ }^{12}$ Depois, fechou-se imediatamente o orifício de oclusão e foi solicitada uma inspiração, tão forte quanto o indivíduo pudesse conseguir, após dois segundos de força sustentada, e terminando a manobra, retirou-se o bocal. No procedimento de medida da pressão expiratória máxima, repetiram-se os três primeiros passos e, a partir desse ponto, solicitou-se uma inspiração mais completa possível ao nível de CPT, também solicitando ao paciente que fizesse um gesto indicativo nesse momento. Em seguida, fechou-se imediatamente o orifício de oclusão e solicitou-se expiração máxima com sustentação por dois segundos. ${ }^{12}$

O indivíduo foi orientado em relação à técnica antes da execução da mesma, fazendo duas ou três manobras de aprendizagem antes de se efetuarem as medidas. Realizaram-se de três a cinco manobras aceitáveis (sem vazamento de ar e sustentando por, no mínimo, um segundo), sendo o intervalo de tempo entre as técnicas de manovacuometria de um minuto. ${ }^{12}$ Para o registro dos resultados, foi considerada a de maior valor. Os dados ficaram armazenados na ficha de avaliação pulmonar, e foram ana- 
lisados e comparados entre os voluntários fumantes e os não-fumantes. Todos os testes foram efetuados por um mesmo examinador, respeitando-se os protocolos preestabelecidos e os dados anotados em ficha de avaliação padrão.

\section{Processamento e avaliação dos dados}

$\mathrm{Na}$ amostra total, em relação ao questionário de hábito tabágico $(\mathrm{n}=1071)$, os resultados das variáveis foram resumidos e apresentados em tabelas, por número de indivíduos de cada categoria, por frequências absolutas e por porcentuais.

As variáveis da capacidade respiratória e a comparação entre os grupos tabagista e não tabagista foram resumidas por meio de número de indivíduos em cada grupo, média, desvio-padrão (DP), valor mínimo e máximo. Para o estudo das variáveis quantitativas, após realizado o teste de normalidade dos dados, utilizou-se o teste $t$ de Student para grupos independentes, segundo o sexo e o hábito de fumar. ${ }^{14,15}$ Empregou-se para os cálculos e análise o software SPSS 21.0, 2013, IBM Incorporation, USA. Adotou-se o nível de $5 \%$ de probabilidade para a rejeição da hipótese de nulidade em todos os testes.

\section{RESUltados}

Foram preenchidos 1.071 questionários, sendo 739 mulheres e 332 homens. As características gerais dos voluntários estão resumidas na Tabela 1.

Tabela 1 - Características gerais dos voluntários fumantes e não-fumantes, segundo sexo, hábito de fumar e conhecimento sobre as doenças associadas ao tabagismo.

\begin{tabular}{llll}
\hline VARIÁVEIS & & $(\mathrm{n})$ & $\%$ \\
\hline \multirow{2}{*}{ Sexo } & Masculino & 332,0 & 31,0 \\
& Feminino & 739,0 & 69,0 \\
\hline \multirow{2}{*}{ Tabagismo } & Sim & 161,0 & 15,0 \\
& Não & 910,0 & 85,0 \\
\hline \multirow{2}{*}{ Frequência do vício } & Festas & $95,0,0$ & 8,9 \\
\hline \multirow{2}{*}{ Sensibilização por campanhas contra o taba- } & Diariamente & 66,0 & 6,2 \\
gismo & Não & 564,0 & 52,7 \\
\multirow{2}{*}{ Influência para o início do tabagismo } & Família & 507,0 & 47,3 \\
& Amigos & 27,0 & 2,5 \\
\multirow{2}{*}{ Preferência de consumo } & Propagandas & 25,0 & 35,1 \\
\hline \multirow{2}{*}{ Conhece as doenças causadas pelo tabagismo? } & Sim & 13.0 & 2,3 \\
\hline \multirow{2}{*}{ Conhece quais doenças são causadas pelo ta- } & Não & 148,0 & 1,2 \\
bagismo? & Câncer & 2,0 & 13,8 \\
\hline & Impotência sexual & 1352,0 & 99,8 \\
& Bronquite & 96,0 & 0,2 \\
\hline
\end{tabular}


Observou-se a incidência de 15\% de estudantes tabagistas que fumavam diariamente ou em festas (eventualmente). A maioria dos estudantes tabagistas respondeu negativamente quando eles foram perguntados se eram sensibilizados a cessar a utilização do tabaco pelas campanhas nacionais do governo. Os amigos tiveram maior influência para o início do vício, sendo que muitos relataram ter experimentado o cigarro por intermédio destes. É importante ressaltar que todos os estudantes que possuíam esse vício conheciam as doenças decorrentes do hábito de fumar e, mesmo sabendo dos malefícios do tabaco, não diminuíram ou cessaram esse vício.

A comparação entre o sexo com as variáveis idade, início do tabagismo e número de cigarros por dia está descrita na Tabela 2. Observou-se resultado significante entre sexo e o número de cigarros por dia, sendo os homens com maior consumo quando comparados com as mulheres.

Tabela 2 - Valores obtidos na comparação entre sexo e as variáveis idade, início do tabagismo e número de cigarro por dia.

\begin{tabular}{llllll}
\hline Variáveis & Sexo & $\mathrm{n}$ & Média & $\mathrm{DP}$ & $\mathrm{p}$ (teste $\mathrm{t}$ ) \\
\hline \multirow{2}{*}{ Idade } & Masculino & 332,0 & 21,2 & 2,1 & \multirow{2}{*}{0,396} \\
& Feminino & 739,0 & 21,1 & 1,9 & \\
\hline \multirow{2}{*}{ Idade que experimentou } & Masculino & 159,0 & 15,7 & 2,6 & \multirow{2}{*}{0,086} \\
\hline \multirow{2}{*}{$\begin{array}{l}\text { Número de cigarro por dia no grupo } \\
\text { tabagista }\end{array}$} & Feminino & 269,0 & 16,2 & 3,0 & \multirow{2}{*}{0} \\
\hline
\end{tabular}

${ }^{\star}$ Diferença significativa

A Tabela 3 mostra os valores obtidos nos testes respiratórios, segundo o hábito de fumar. Verificou-se que o resultado do teste $t$ foi significante para PImáx, PEmáx, CRaxilar, CRmamilar e CR10 ${ }^{a}$ costela, sendo os melhores valores no grupo não tabagista.

Tabela 3 - Valores obtidos na comparação entre os grupos tabagista e não tabagista e as variáveis da capacidade respiratória.

\begin{tabular}{|c|c|c|c|c|c|}
\hline Variáveis & Tabagista & $\mathbf{N}$ & Média & DP & $\mathrm{p}$ \\
\hline \multirow{2}{*}{ PImáx (cmH2O) } & Sim & 20 & 92,7 & 11,1 & \multirow{2}{*}{$0,001^{*}$} \\
\hline & Não & 20 & 115,3 & 7,0 & \\
\hline \multirow{2}{*}{ PEmáx (cmH2O) } & Sim & 20 & 95,0 & 7,2 & \multirow{2}{*}{$0,001^{*}$} \\
\hline & Não & 20 & 108,4 & 8,6 & \\
\hline \multirow{2}{*}{ CR axilar $(\mathrm{cm})$} & Sim & 20 & 5,9 & 1,0 & \multirow[b]{2}{*}{$0,001^{*}$} \\
\hline & Não & 20 & 7,1 & 0,7 & \\
\hline \multirow{2}{*}{ CR mamilar $(\mathrm{cm})$} & Sim & 20 & 6,1 & 1,1 & \multirow{2}{*}{$0,001^{*}$} \\
\hline & Não & 20 & 7,2 & 0,7 & \\
\hline CR $10^{\mathrm{a}}$ costela $(\mathrm{cm})$ & Sim & 20 & 6,2 & 0,9 & $0,001^{*}$ \\
\hline
\end{tabular}

PImáx: Pressão Inspiratória Máxima; PEmáx: Pressão Expiratória Máxima; CR, coeficiente respiratório; DP, desvio padrão; ${ }^{\star} \mathrm{p}<0,05$ (Teste $\mathrm{t}$ ) 
Segundo o sexo, observou-se resultado não significante para PImáx e PEmáx (Tabela 4). Para a variável CR axilar, o resultado foi significante com os homens apresentando maiores valores do que as mulheres. Para as variáveis $\mathrm{CR}$ mamilar e $\mathrm{CR} 10^{\mathrm{a}}$ costela, o resultado foi estatisticamente significante, com maiores valores para os homens, em relação às mulheres.

Tabela 4 - Comparação entre o sexo, masculino e feminino nas variáveis da capacidade respiratória.

\begin{tabular}{|c|c|c|c|c|c|}
\hline Variáveis & Sexo & $\mathrm{n}$ & Média & DP & $\mathrm{p}$ \\
\hline \multirow{2}{*}{ PImáx (cmH2O) } & Masculino & 20 & 105,8 & 15,4 & \multirow{2}{*}{0,444} \\
\hline & Feminino & 20 & 102,2 & 14,0 & \\
\hline \multirow{2}{*}{ PEmáx (cmH2O) } & Masculino & 20 & 103,7 & 10,0 & \multirow{2}{*}{0,221} \\
\hline & Feminino & 20 & 99,7 & 10,6 & \\
\hline \multirow{2}{*}{ CR axilar $(\mathrm{cm})$} & Masculino & 20 & 6,9 & 1,0 & \multirow{2}{*}{0,013} \\
\hline & Feminino & 20 & 6,1 & 0,9 & \\
\hline \multirow{2}{*}{ CR mamilar (cm) } & Masculino & 20 & 7,2 & 1,0 & \multirow{2}{*}{$0,001^{\star}$} \\
\hline & Feminino & 20 & 6,1 & 0,9 & \\
\hline \multirow{2}{*}{ CR $10^{\mathrm{a}}$ costela $(\mathrm{cm})$} & Masculino & 20 & 7,3 & 0,9 & \multirow{2}{*}{$0,001^{*}$} \\
\hline & Feminino & 20 & 6,3 & 0,8 & \\
\hline
\end{tabular}

PImáx: Pressão Inspiratória Máxima; PEmáx: Pressão Expiratória Máxima; CR, coeficiente respiratório; DP, desvio padrão; ${ }^{\star} \mathrm{p}<0,05$ (Teste $\mathrm{t}$ )

\section{DIscussão}

Dos 1.071 estudantes entrevistados na pesquisa, $15 \%$ relataram fazer uso diário ou eventual do tabaco. Esse achado concorda com o que foi descrito por outros autores, ${ }^{10}$ que apontaram prevalência de tabagismo de 15,4\% (182) entre universitários e, em outro estudo semelhante, numa população de 142 acadêmicos, $14,1 \%$ eram tabagistas. ${ }^{7}$

Pode-se inferir que tal situação se deve à maior conscientização dos jovens em relação aos hábitos que promovem boa saúde, tanto física quanto mental, o que se consegue pela boa alimentação, pela atividade física e pelo desuso do tabaco. Em outro estudo, ${ }^{15}$ foi apontada correlação $20 \%$ negativa entre tabagismo e história familiar, sugerin- do que os indivíduos que apresentam história familiar de doenças cardiovasculares tendem, em geral, a serem não tabagistas.

A presente pesquisa demonstrou também que aqueles que experimentaram o tabaco tiveram contato com o cigarro quando mais novos, devido à influência dos amigos. Corroborando com esse achado, foi descrito em um estudo que a maioria dos entrevistados começou a consumir o tabaco com idade inferior a 20 anos e que o principal motivo que os levou a fumar foi a influência do grupo social em que estavam inseridos. ${ }^{6,16}$ Esse estudo revelou que o fumar é prevalente em festas e está associado ao consumo de bebidas.

A literatura aponta relação bidirecional entre essas variáveis. Estudos como o de Gar- 
cia et al. ${ }^{18}$ (2009), realizado entre 492 acadêmicos da área da saúde da universidade Estadual da Paraíba (UEPB), e Rodrigues Junior et al. ${ }^{14}$ (2009), avaliando o tabagismo em instituições de nível superior, confirmaram essa relação. O consumo precoce e a influência do grupo em que estão inseridos nos alertam para a importância de se compreender as características de consumo e o perfil dessa população, visando garantir o acesso à informação e aprimorar programas de prevenção do tabagismo nas instituições de ensino superior.

Outra característica encontrada nos jovens consumidores de tabaco é a preocupação prioritária com a marca do produto (13,8\% dos tabagistas) do que com o preço da mercadoria (1,2\% dos entrevistados tabagistas), estudos nacionais ${ }^{6,10}$ e internacionais ${ }^{5,19}$ concordam com o presente achado. Esse fator chama a atenção em relação à influência da mídia, especialmente nesse período da juventude que é propício para a indústria do tabaco investir em propagandas no sentido de estimular o vício. Vale notar que, apoiado por esses resultados já consolidados pelas pesquisas sobre o tabaco, foram criadas leis que regulamentam a propaganda e restringem o uso do tabaco em locais públicos.

Apesar de os estudantes estarem cientes dos malefícios do cigarro, as campanhas do governo não foram capazes de sensibilizar totalmente quanto à cessação do tabagismo. Estudos como o de Rodrigues et al. ${ }^{17}$ (2008) e Silva et al. ${ }^{20}$ (2008) também mostraram esses dados.

Na comparação entre os sexos e o número de cigarros que os estudantes consomem por dia, o masculino possui média maior em relação ao feminino (6,1 versus 4,6 cigarros/ dia, $\mathrm{p}<0,001)$. Em estudos de Brandão et al. ${ }^{21}$ (2008), foi observada uma baixa proporção de fumantes do sexo feminino quan- do comparado com o sexo masculino, assim como mostra o presente estudo. Um achado importante neste estudo revelou que quase a totalidade da população em evidência mostra conhecimento em relação às doenças e aos malefícios do tabaco $(99,8 \%$ da população total), porém, apesar desse conhecimento, ainda possuem o vício.

Os resultados de PIMáx e PEMáx obtidos no presente estudo são condizentes com os valores de referência para a população brasileira, ${ }^{10}$ porém na comparação entre os grupos tabagista e não tabagista, as variáveis da capacidade respiratória (PImax, PEmax e cirtometrias) apresentaram-se com valores menores no grupo tabagista, sendo este um resultado importante devido ao impacto do uso do tabaco no sistema respiratório, mesmo sendo de forma precoce.

Quanto ao sexo, estudos de Matecki et al. $^{22}$ (2003) e Harik-Khan et al. ${ }^{23}$ (1998) demonstram que a força muscular respiratória é maior no masculino que no feminino, corroborando com o achado do presente estudo. Em relação à mobilidade torácica, estudo de Willrich et al. ${ }^{24}$ (2002) mostrou que as médias dos valores de mobilidade torácica foram maiores no sexo masculino, apontando um dado semelhante ao presente estudo.

Sendo assim, tais resultados nos levam a refletir sobre a necessidade de se desenvolverem e estimular em abordagens interdisciplinares, buscando-se as conexões metodológicas entre as diversas áreas para se compreender o processo e se aumentar a eficácia dos programas de controle do uso do tabaco, além de se investir na implementação de estudos preventivos.

Com base nessas necessidades, devem-se desenvolver programas educacionais de prevenção em ensino fundamental e médio, pois foi observado no estudo que a 
idade de início desse hábito dá-se na adolescência. Ressalta-se também, a presença de restrição respiratória dos fumantes participantes do estudo, o que mostra que a cessação do tabaco em longo prazo pode prevenir doenças respiratórias.

\section{CONSIDERAÇões FinaIS}

Foram identificados $15 \%$ de usuários de tabaco entre os estudantes da UNIFAL-MG. Em relação à capacidade respiratória desses estudantes tabagistas, quando comparada com não tabagistas, foram evidenciados menores valores de capacidade respiratória nos parâmetros de PImax, PEmax e cirtometrias. Esses resultados levam, portanto, a refletir sobre a necessidade de se investir na implementação de estudos preventivos que envolvam elementos objetivos na discussão dos riscos consequentes ao vício do tabaco nas diversas áreas do conhecimento, principalmente na área da saúde.

\section{REFERÊNCIAS}

1. World Health Organization - WHO Report on the Global Tobacco Epidemic 2015. The MPower Pachage. Geneve: WHO, 2015.

2. Mendes ACR, Toscano CM, Barcellos RMS, Ribeiro ALP, Ritzel JB, Cunha VS, Duncan BB. Custos do Programa de Tratamento do Tabagismo no Brasil. Rev Saúde Pública. 2016; 50: $1-12$.

3. Brasil. Instituto Nacional do Câncer. Ação Global para o controle do Tabaco. Primeiro Tratado Internacional de Saúde Pública. Brasília: Ministério da Saúde; 2002.

4. Rigotti NA, Lee JE, Wechsler H. US College students use of tobacco products: results of nature survey. JAMA 2000; 284: 699-705.

5. Urrutia-Pereira M., Oliano VJ, Aranda CS, Mallol J., Solé D. Prevalence and factors associated with smoking among adolescents. J Pediatr. 2017;93(3):230-237.

6. Andrade APA, Bernardo ACC, Viegas CAA, Ferreira DBL, Gomes TC, Sales MR. Prevalência e características do tabagismo em jovens da Universidade de Brasília. J Bras Pneumol. 2006; 32: 23-28.

7. Heinisch RH, Zukowski CN, Heinisch LMM. Fatores de risco cardiovascular em acadêmicos de medicina. ACM. Arq. Catarin. Med. 2007; 36(1): 76-84.

8. Carvajal S., Wiatrek DE, Evans RI, Knee CR, Nash SG. Psychosocial determinants on the onset of escalation of smoking: cross sectional and prospective findings in multiethinic middle school samples. J Adolesc Health 2000; 27: 255-65.

9. Simantov E., Schoen C., Klein JD. Health compromising behaviours: why do adolescents smoke or drink? Identifying underlying risk and protective factors. Arch Pediatr Adolesc Med 2000; 154: 1.025-33.

10. Miranda JS, Almeida JB, Marques SFG. Prevalência e fatores associados ao tabagismo em uma unidade universitária. Enfermagem Brasil 2009; 8: 266-271.

11. Brasil. Instituto Nacional do Câncer (INCA). Ação Global para o controle do Tabaco. Primeiro Tratado Internacional de Saúde Pública. Brasília: Ministério da Saúde; 2002.

12. Britto RR, Brant TCS, Parreira VF. Recursos manuais e instrumentais em fisioterapia respiratória, 1. ed. São Paulo: Manole, 2009.

13. Neder JA, Andreoni S., Lerario MC, Nery LE. Reference values for lung function tests. II. Maximal respiratory pressures and voluntary ventilation. Braz J Med Biol Res. 1999; 32, 6: 719-27. 
14. Rodrigues Junior JC, Ferraz SMR, Bruno RX. Prevalência e perfil dos tabagistas universitários em ingressantes de uma instituição de ensino superior. Pulmão RJ 2009; 18: 14-18.

15. Carvalho AA. Semiologia em reabilitação. São Paulo: Atheneu, 1994.

16. Morais CAS, Oliveira SHV, Brandão FC, Gomes IF, Lima LM. Fatores de risco cardiovascular em estudantes de graduação da Universidade Federal de Viçosa-MG. J Health Sci Inst. 2011, 29, 4: 261-4.

17. 17- Rodrigues ESR, Cheik NC, Mayer AF. Nível de atividade física e tabagismo entre universitários. Rev Saúde Pública 2008; 42: 672-678.

18. Garcia GFA, Branco ACL, Sarmento DJS, Cavalcanti AL, D’Ávila S., Menezes VA. Tabagismo e fatores associados entre acadêmicos de odontologia. RFO 2009; 14: 92-98.

19. Haddad LG, Malak MZ. Smoking habits and attitudes towards smoking among university students in Jordan. Int J Nurs Stud. 2002; 39: 793-802.

20. Silva AO, Gaspar MFM, Paredes MAS, Tura LFR, Jesuíno JC. Tabaco e saúde no olhar do estudante universitário. Rev Bras Enferm 2008; 61: 423-427.

21. Brandão MP, Pimentel FL, Silva CC, Cardoso MF. Factores de risco cardiovascular numa população universitária portuguesa. Rev Port Cardiol 2008; 27: 7-25.

22. Matecki S., Prioux J., Jaber S., Hayot M., Prefaut C., Ramonatxo M. Respiratory pressures in boys from 11-17 years old: a semilongitudinal study. Pediatr Pulmonol. 2003; 35: 368-74.

23. Harik-Khan RI, Wise RA, Fozard JL. Determinants of maximal inspiratory pressure. The Baltimore Longitudinal Study of Aging. Am J Respir Crit Care Med. 1998; 158: 1.459-64.

24. Willrich FR, et al. Análise da cirtometria torácica em indivíduos saudáveis na faixa etária de 8 a 10 anos. Rev Bras Fisiot. Supp., p. 83, 2002.

\section{Dados dos Autores}

\section{Monique Silva Rezende}

Doutoranda do Programa de Reabilitação e Desempenho Funcional da Universidade de São Paulo. Alfenas/MG - Brasil. monique_rezende@hotmail.com

\section{Gabriel Casare Ogasawara}

Graduado em Fisioterapia pela Universidade Federal de Alfenas. Alfenas/MG - Brasil. gabriel_casare@hotmail.com

\section{Juliana Bassalobre Carvalho Borges}

Doutora em Bases Gerais da Cirurgia pela Faculdade de Medicina de Botucatu. Alfenas/ MG - Brasil. juliana.borges@unifal-mg.edu.br

\section{Cristiane Costa Fonseca}

Doutoranda em Cirurgia Translacional pela Universidade Federal de São Paulo. Alfenas/ MG - Brasil.ccricf@yahoo.com.br

\section{Leonardo César Carvalho}

Doutor em Ciências Médicas pela Universidade de São Paulo. Alfenas/MG - Brasil. leounifal@gmail.com 


\section{Denise Hollanda IUnes}

Doutora em Ciências Médicas pela Universidade de São Paulo. Alfenas/MG - Brasil. deniseiunes@unifal-mg.edu.br

\section{Carmélia Bomfim Jacó Rocha}

Doutora pelo departamento de Cirurgia da Universidade de Campinas. Alfenas/MG - Brasil.carmelia.rocha@unifal-mg.edu.br

Submetido em: 06-10-2016

Aceito em: 22-06-2017 